\title{
Erratum: Enhanced Casimir effect for doped graphene [Phys. Rev. B 93, 075414 (2016)]
}

M. Bordag, I. Fialkovskiy, and D. Vassilevich (Received 7 March 2017; published 24 March 2017)

DOI: 10.1103/PhysRevB.95.119905

Due to the last-minute change of notation in Eq. (6) the expressions for $\Delta \Pi_{\mathrm{xx}}$ and $M_{t r}$ given in our paper are incorrect. They must read

$$
\Delta \Pi_{\mathrm{xx}}=\frac{8 \alpha}{v_{F}^{2}} \int_{m}^{\infty} d \varkappa\left(1+\operatorname{Re} \frac{M_{\mathrm{xx}}}{\sqrt{Q^{2}-4 v_{F}^{2} p_{\|}^{2}\left(\varkappa^{2}-m^{2}\right)}}\right) \Xi(\varkappa), \quad M_{t r}=-\tilde{p}^{2}+4 \varkappa\left(1-v_{F}^{2}\right)\left(i p_{4}+\varkappa\right)+4 v_{F}^{2} m^{2} .
$$

For the same reason Eq. (8) should read

$$
\Delta \Pi_{t r}(p)=8 \alpha\left(\frac{\mu}{v_{F}^{2}}-\operatorname{Im}\left[\frac{\tilde{p}^{2}+p^{2}}{4 \tilde{p}} \log \left(x+\sqrt{x^{2}+1}\right)+\frac{\left(1-v_{F}^{2}\right) p_{\|}^{2}}{4 \tilde{p}} x \sqrt{x^{2}+1}\right]\right) .
$$

The results of the numerical evaluation given in the paper are not influenced by these misprints.

The authors are grateful to Prof. Galina Klimchitskaya for calling our attention to the misprints. 